\title{
UNIFORM METHODS TO ESTABLISH POINCARÉ TYPE LINEARIZATION THEOREMS
}

\author{
HAO WU \\ This paper is dedicated to celebrate Jaume Llibre's 60th birthday. \\ He inspires me a lot in my math study.
}

\begin{abstract}
We find a uniform method to establish Poincaré type linearization theorems for regular systems including classical autonomous, random and almost periodic ones via modified majorant norm methods.
\end{abstract}

2010 Mathematics Subject Classification: 34C20, 37H10.

Key words: Normal form, random dynamical system, almost periodic system, majorant norm method.

\section{Introduction and statement of the main results}

Normal form theory is used to simplify ordinary differential equations through changes of variables and it was initiated by Poincaré [19]. Inspired by the work of Bibikov [4], Sternberg [20], Chen [5], Takens [21], and many other mathematicians, nowadays it plays an important role in the study of bifurcation and stability, among others. In recent years serious results have been achieved with the development of new techniques including the way to calculate the holomorphy for analytic admissible autonomous systems in $[\mathbf{3}]$, homotopy methods for random dynamical systems in $[\mathbf{1 6}, \mathbf{1 7}]$, the application of exponential dichotomy spectra theory for differential and difference non-autonomous systems in [15] and [22], correction function methods for the analytic linearization of Fuchsian systems in $[\mathbf{7}, \mathbf{8}]$, majorant norm methods for the new proof of the classical Poincaré Theorem in [13], etc.

In general the construction of normal forms reflects deeply on two basic aspects, one is the dynamical behavior of the linear parts especially for non-autonomous ones, the other is the study of normal form operators in convenient functional spaces. Due to such difficulties for generic dynamical systems, here we restrict our attention to the simplest ones, 
which are with Lyapunov 'regular' linear parts, i.e. with fundamental matrices $X(t)$ of the form

$$
X(t)=Z(t) e^{\operatorname{diag}\left(\alpha_{1}, \ldots, \alpha_{d}\right) t},
$$

and belong to the Poincaré domain, i.e. Lyapunov exponents are of the same signs. See [1] for more details about the regularity. Therefore, we manage to detect a uniform way to build Poincaré type linearization theorems for such systems. The methods used here stem from the technique of controlling sub-exponential unbounded nonlinearities in $[\mathbf{1 6}, \mathbf{1 7}]$, the applications of admissible conditions in [3] and classical majorant norm methods in [12].

In brief, first of all we establish an analytical linearization criterion for systems with diagonal linear parts but sub-exponential unbounded nonlinearities. Consider the following non-autonomous differential systems

$$
\frac{d x}{d t}=\Lambda x+f(t, x) .
$$

Here $\Lambda=\operatorname{diag}\left(\lambda_{1}, \ldots, \lambda_{d}\right), f(t, x)$ is continuous in $t$ and analytic in $x$ at $x=0$ for every fixed $t, f(t, 0) \equiv 0$ and $D_{x} f(t, 0) \equiv 0$. As usual $D_{x} f$ denotes the Jacobian matrix of $f$ with respect to the variable $x$. Moreover, we say $f(t, x)=\sum_{l=1}^{d} \sum_{|k| \geq 2} f_{k}^{l}(t) x^{k} e_{l} \in \mathcal{C}_{k \delta}(\Lambda)$, provided that for all the monomials $f_{k}^{l}(t) x^{k} e_{l}$ satisfying $f_{k}^{l}(t) \not \equiv 0$ there exists $\delta>0$ such that $\langle k, \lambda\rangle-\lambda_{l} \leq-|k| \delta$ or $\langle k, \lambda\rangle-\lambda_{l} \geq|k| \delta$. Here $e_{l}$ is the unit vector with the $l$-th component $1,|k|=\sum_{i=1}^{d} k_{i} \geq 2$ and $\langle k, \lambda\rangle=$ $\sum_{i=1}^{d} k_{i} \lambda_{i}$. Specially, we note that this definition is always valid whatever $f$ depends on $t$ or not.

Theorem 1. Let $\rho(t)$ and $M(t)$ be two positive continuous functions. Assume that $f(t, x) \in \mathcal{C}_{k \delta}(\Lambda)$ in system (1) and

$$
\max _{|x| \leq \rho(t)}|f(t, x)| \leq M(t), \quad t \in \mathbb{R} .
$$

If there exists a positive function $\kappa(t)$ satisfying the following conditions:

$(\mathrm{C} 1) \kappa(t) \leq \min \{1 /(C(d) M(t)), \rho(t) / 2,1\}$, where $C(d)=d \sum_{|k| \geq 0}(1 / 2)^{|k|}$ is a constant, and

(C2) there exists a positive constant $\tau<\delta$ such that

$$
\frac{\kappa(t+h)}{\kappa(t)} \leq e^{\tau|h|}, \quad t, h \in \mathbb{R},
$$


then there exist coordinates substitutions $x=y+h(t, y)$, which are analytic in the domain $\left\{(t, x)|| x \mid \leq r_{0} \kappa(t)\right\}$ for a fixed $t$ and some $r_{0}>0$, changing system (1) into the linear system $\dot{y}=\Lambda y$. Moreover, if the functions $\rho(t)$ and $M(t)$ additionally satisfy

$$
\lim _{|t| \rightarrow \infty} \frac{1}{|t|} \ln ^{+} M(t)=0, \quad \lim _{|t| \rightarrow \infty} \frac{1}{|t|} \ln ^{-} \rho(t)=0,
$$

where $\ln ^{+} x=\max \{\ln x, 0\}$ and $\ln ^{-} x=\min \{\ln x, 0\}$, then for every fixed $y$ the function $h(t, y)$ admits the limit

$$
\lim _{|t| \rightarrow \infty} \frac{1}{|t|} \ln |h(t, y)|=0 .
$$

Next, as applications, we study the analytic conjugate problems for autonomous, random and almost periodic dynamical systems in the same frame. By doing a linear non-autonomous change, we force those systems into the form (1). In general, that transformation may destroy the original autonomous, random, and almost periodic structures. So, technically saying, the proofs of such classical theorems can be split into two parts. One is the convergence of the transformation, which shall be confirmed by Theorem 1; the other is the formal preservation due to their corresponding structures.

Consider the analytic differential system

$$
\dot{x}=A x+f(x),
$$

where $A=D+N$ is in the Jordan normal form with the diagonal part $D$ and the nilpotent part $N, f(0)=0, D_{x} f(0)=0$ and $f$ is analytic at the origin.

Theorem 2. Assume that $f \in \mathcal{C}_{k \delta}(D)$ in system (2). Then there exist coordinates substitutions $x=y+h(y)$, which are analytic at the origin, changing system (2) into the linear system $\dot{y}=A y$.

This result is new because of the existence of nilpotent parts, while the diagonal case was discussed in $[\mathbf{3}]$. Moreover, we specially note that our conditions cannot be weakened if $N \neq 0$. For more details see [11].

Let $(\Omega, \mathcal{F}, \mathbb{P})$ be a probability space and we use the notation $\dot{x}_{t}=$ $\frac{d x_{t}}{d t}$. Then consider the random system associated with the dynamical system $\left(\Omega, \mathcal{F}, \mathbb{P},\left(\theta_{t}\right)_{t \in \mathbb{R}}\right)$ defined by the differential equation

$$
\dot{x}_{t}=A\left(\theta_{t} \omega\right) x_{t}+f\left(\theta_{t} \omega, x\right)=F\left(\theta_{t} \omega, x\right),
$$

under the following hypotheses: 
(i) The condition of the Multiplicative Ergodic Theorem is satisfied, i.e.

$$
\alpha^{ \pm}(\omega):=\sup _{0 \leq t \leq 1} \ln ^{+}\left\|\Phi(\omega, t)^{ \pm}\right\| \in L^{1}(\Omega) .
$$

(ii) The function $F\left(\theta_{t} \omega, x\right)$ is analytic in $x$ for a fixed $t$ and there exist $\rho(\omega)$ tempered from below and $M(\omega)$ tempered from above such that

$$
\max _{|x| \leq \rho\left(\theta_{t} \omega\right)}\left|f\left(\theta_{t} \omega, x\right)\right|=M\left(\theta_{t} \omega\right)<\infty .
$$

(iii) $F\left(\theta_{t} \omega, x\right), \rho\left(\theta_{t} \omega\right)$ and $M\left(\theta_{t} \omega\right)$ are all continuous in $t$ for $\omega \mathbb{P}$ a.e.

As usual a random variable $R: \Omega \rightarrow(0, \infty)$ is called tempered with respect to a metric dynamical system $\theta_{t}$ if

$$
\lim _{|t| \rightarrow \infty} \frac{1}{t} \ln R\left(\theta_{t} \omega\right)=0, \mathbb{P} \text { a.e., }
$$

$R: \Omega \rightarrow[0, \infty)$ is called tempered from above if

$$
\lim _{|t| \rightarrow \infty} \frac{1}{t} \ln ^{+} R\left(\theta_{t} \omega\right)=0, \mathbb{P} \text { a.e., }
$$

and $R: \Omega \rightarrow(0, \infty)$ is called tempered from below if $1 / R$ is tempered from above. Furthermore, Lyapunov exponents $\gamma(\omega)=\left(\gamma_{1}(\omega), \ldots, \gamma_{d}(\omega)\right)$ are called non-resonant if $\langle k, \gamma(\omega)\rangle \neq \gamma_{l}(\omega)$ for all $1 \leq l \leq d$ and $|k| \geq 2$. Then we provide a new proof of the Poincaré linearization theorem for random dynamical systems. See [16] for another proof by homotopy methods.

Theorem 3. Under hypotheses (i), (ii), and (iii) if the Lyapunov exponents $\gamma(\omega)=\left(\gamma_{1}(\omega), \ldots, \gamma_{d}(\omega)\right)$ have the same signs and are nonresonant, then there exist coordinates substitutions $x_{t}=y_{t}+h\left(\theta_{t} \omega, y_{t}\right)$, which are analytic in some tempered ball, changing system (3) into the linear system $\dot{y}_{t}=A\left(\theta_{t} \omega\right) y_{t}$.

Finally, we show that such math model (Theorem 1) is valid for those systems, which can apply averaging methods. More precisely, suppose that $U$ is a non-empty set, $x \in U \subseteq \mathbb{C}^{d}$ and $\varepsilon \geq 0$ is a real parameter, then we say $f \in \mathcal{A} \mathcal{F}([0, \infty), U)$, if

(i) the function $f: \mathbb{R} \times[0, \infty) \times U \rightarrow \mathbb{C}^{d}$ is continuous,

(ii) $f(t, \varepsilon, x)$ is almost periodic in $t$ uniformly with respect to $x$ in compact sets of $U$ for each fixed $\varepsilon$,

(iii) $f(t, \varepsilon, x)$ is analytic with respect to $x \in U$ for every fixed $t$ and $\varepsilon$,

(iv) $f(t, \varepsilon, x) \rightarrow f(t, 0, x)$ as $\varepsilon \rightarrow 0$ uniformly for $t \in \mathbb{R}$ and $x$ in compact sets. 
We associate the system of differential equations

$$
\dot{x}:=\frac{d x}{d t}=\varepsilon f(t, \varepsilon, x),
$$

with the averaged system

$$
\dot{x}=\varepsilon f_{0}(x),
$$

where

$$
f_{0}(x)=\lim _{T \rightarrow \infty} \frac{1}{T} \int_{0}^{T} f(t, 0, x) d t .
$$

By the classical averaging theorem (see $[\mathbf{9}, \mathbf{1 0}]$ ), if $x_{0} \in U$ is a hyperbolic singular point of system (5), then system (4) has an almost periodic solution $x^{*}(t, \varepsilon) \rightarrow x_{0}$ uniformly as $\varepsilon \rightarrow 0$.

Let $U_{\delta}=\left\{x \in \mathbb{C}^{d}:\|x\| \leq \delta\right\}$ and $A$ be a $d$-square matrix. Set $\lambda(A)=\left(\lambda_{1}, \ldots, \lambda_{d}\right)$ be the eigenvalues of $A$. Denote by Re the real part of a complex number. Without loss of generality, we can assume that $\operatorname{Re} \lambda_{1} \leq \cdots \leq \operatorname{Re} \lambda_{d}$. We say that the eigenvalues $\lambda(A)$ are in the Poincaré domain if $\operatorname{Re} \lambda_{d}<0$ or $\operatorname{Re} \lambda_{1}>0$. The following conditions are called resonant conditions:

$$
\sum_{i=1}^{d} k_{i} \operatorname{Re} \lambda_{i}-\operatorname{Re} \lambda_{j}=0 .
$$

Here $k=\left(k_{1}, \ldots, k_{d}\right) \in \mathbb{Z}_{+}^{d}$ and $j=1, \ldots, d$. As usual, $\mathbb{Z}_{+}$denotes the set of non-negative integers. We say that $A$ is non-resonant, if $\lambda(A)$ possess non-resonant conditions for every $|k|=\sum_{i=1}^{d} k_{i} \geq 2$ and $j=1, \ldots, d$.

System (4), with the change of variables $x \mapsto y$ given by

$$
x=y+x^{*}(t, \varepsilon),
$$

becomes

$$
\dot{y}=\varepsilon F(t, \varepsilon, y) .
$$

Here $F(t, \varepsilon, 0) \equiv 0$ and $F(t, \varepsilon, y)=f\left(t, \varepsilon, y+x^{*}(t, \varepsilon)\right)-f\left(t, \varepsilon, x^{*}(t, \varepsilon)\right)$ satisfies the same conditions as the function $f(t, \varepsilon, x)$. As usual, $D_{y}$ denotes the Jacobian matrix with respect to $y$ and $m(f)$ is remarked as the module of an almost periodic function $f$.

Theorem 4. Let $A=D_{x} f_{0}\left(x_{0}\right)$. If $\lambda(A)$ is in the Poincaré domain and non-resonant, then there exist $\varepsilon_{0}>0$ and $\delta>0$ independent of $\varepsilon$ such that for $0<\varepsilon \leq \varepsilon_{0}$ under the change of coordinates $y=z+w(t, \varepsilon, z)$, where $w \in \mathcal{A F}\left(\left[0, \varepsilon_{0}\right), U_{\delta}\right), D_{z} w(t, \varepsilon, 0) \rightarrow 0$ uniformly for $t \in \mathbb{R}$ as $\varepsilon \rightarrow 0$ and $m(w) \subset m(F)$, system (6) becomes

$$
\dot{z}=\varepsilon D_{y} F(t, \varepsilon, 0) z \text {. }
$$


In general, normal forms are studied one by one for non-autonomous systems. However, when we study the invalidity of Seigel type theorems for random diffeomorphisms, it is shown that there actually exist some common laws for general ones. Therefore, we extend the criterion $[\mathbf{1 4}$, Theorem 3.4] to Theorem 1 for more systems, whose linear parts are near the diagonal constant ones. Then using it, we provide an extension of the results in [3] and new proofs of partial results in [15] and [16]. Moreover, by playing tricks in $[\mathbf{1 3}]$, Poincaré analytic polynomial normal forms also can be achieved. Additionally, we specially note that our definitions of the Poincaré domain for systems (3) and (6) stem from the one of real autonomous systems, because those systems are non-autonomous and the time variable is real.

The reminder parts are organized as follows. In $\S 2$ basic introductions and fundamental properties are provided for classical majorant norms, random and almost periodic systems. Then Theorem 1, the analytic linearization criterion, is established in $\S 3$, which naturally leads to proofs of Theorem 2, 3 and 4 in $\S 4, \S 5$ and $\S 6$, respectively. And some other applications are also mentioned in $\S 7$.

\section{Preliminaries}

In this part, first we introduce some basic definitions and properties of majorant norms, most of which are from [13]. Next we continue with the background of random dynamical systems and the statement of the Multiplicative Ergodic Theorem from [2]. Finally, we recall some classical results of the exponential dichotomy and almost periodicity from [6].

The majorant operator is the nonlinear operator acting on formal power series by replacing all Taylor coefficients by their absolute values,

$$
\mathcal{M}: \sum_{\alpha \in \mathbb{Z}_{d}^{+}} C_{\alpha} z^{\alpha} \mapsto \sum_{\alpha \in \mathbb{Z}_{d}^{+}}\left|C_{\alpha}\right| z^{\alpha} .
$$

Moreover, the majorant $\rho$-norm is the function on the space of formal power series, given by

$$
\llbracket f \rrbracket_{\rho}=|\mathcal{M} f(\rho, \ldots, \rho)| \leq \infty .
$$

Then we provide the fundamental property of majorant norms, for more details see $[\mathbf{1 3}]$. Let $\mathbb{C}[[x]]$ be the space of formal series with complex coefficients. Given $f$ and $g$ two vector fields in $\mathbb{C}[[x]]$ with positive coefficients, we denote by $f \ll g$ when each coefficient of $f$ is no greater than the corresponding one of $g$. 
Proposition 5. Let $f$ and $g \in \mathbb{C}[[x]]$. Then the following statements hold:

(i) $\mathcal{M}(f g) \ll \mathcal{M} f \cdot \mathcal{M} g$,

(ii) $\mathcal{M}(f \circ g) \ll \mathcal{M} f \circ \mathcal{M} g$.

Proof: The proof follows by a straightforward computation of the coefficients of $f \cdot g$ and $f \circ g$.

Next we give basic concepts of random dynamical systems and related theorems. Let $(\Omega, \mathcal{F}, \mathbb{P})$ be a probability space and $\mathbb{T}$ and $\mathbb{F}$ are $\mathbb{R}$ or $\mathbb{C}$, where one is related to the base space and the other to the bundle space. Moreover, $\mathbb{T}$ is endowed with its Borel $\sigma$-algebra $\mathcal{B}(\mathbb{T})$. We say that a family $\left(\theta_{t}\right)_{t \in \mathbb{T}}$ of mappings from $\Omega$ into itself is called a metric dynamical system if:

(i) $(\omega, t) \rightarrow \theta_{t} \omega$ is $\mathcal{F} \otimes \mathcal{B}(\mathbb{T})$ measurable;

(ii) $\theta_{0}=\operatorname{Id}_{\Omega}$, the identity on $\Omega, \theta_{t+s}=\theta_{t} \circ \theta_{s}$ for all $t, s \in \mathbb{T}$;

(iii) $\theta_{t}$ preserves the probability measure $\mathbb{P}$.

The map

$$
\psi: \mathbb{T} \times \Omega \times \mathbb{F}^{d} \rightarrow \mathbb{F}^{d}, \quad(t, \omega, x) \mapsto \psi(t, \omega, x)
$$

is called a random dynamical system (or a cocycle) on the measurable space $(\omega, \mathcal{F})$ over a metric dynamical system $\left(\Omega, \mathcal{F}, \mathbb{P}, \theta_{t}\right)$ if:

(i) $\psi$ is $\mathcal{B}(\mathbb{T}) \otimes \mathcal{F} \otimes \mathcal{B}\left(\mathbb{C}^{d}\right)$-measurable;

(ii) the map $\psi(t, \omega):=\psi(t, \omega, \cdot): \mathbb{F}^{d} \rightarrow \mathbb{F}^{d}$ forms a cocycle over $\theta_{t}$ :

$$
\begin{array}{ll}
\psi(0, \omega)=\mathrm{Id} & \text { for all } \omega \in \Omega, \text { and } \\
\psi(t+s, \omega)=\psi\left(s, \theta_{t} \omega\right) \circ \psi(t, \omega) & \text { for all } s, t \in \mathbb{T}, \omega \in \Omega .
\end{array}
$$

In addition, a random variable $R: \Omega \rightarrow(0, \infty)$ is called tempered with respect to a metric dynamical system $\theta_{t}$ if

$$
\lim _{|t| \rightarrow \infty} \frac{1}{|t|} \ln R\left(\theta_{t} \omega\right)=0, \mathbb{P} \text { a.e., }
$$

$R: \Omega \rightarrow[0, \infty)$ is called tempered from above if

$$
\lim _{|t| \rightarrow \infty} \frac{1}{|t|} \ln ^{+} R\left(\theta_{t} \omega\right)=0, \mathbb{P} \text { a.e. }
$$

and $R: \Omega \rightarrow(0, \infty)$ is called tempered from below if $1 / R$ is tempered from above. The result below is the celebrated Multiplicative Ergodic Theorem, whose statement comes from Theorem 3.4.11 and Corollary 4.3.12 in $[2]$. 
Lemma 6. Consider the random system associated with the dynamical system $\left(\Omega, \mathcal{F}, \mathbb{P},\left(\theta_{t}\right)_{t \in \mathbb{R}}\right)$

$$
\dot{x}_{t}=A\left(\theta_{t} \omega\right) x_{t},
$$

where $A\left(\theta_{t} \omega\right)$ is continuous in $t \mathbb{P}$ a.e. and the corresponding generated linear cocycle is denoted by $\Phi(\omega, t)$. Assume that

$$
\alpha^{ \pm}(\omega):=\sup _{0 \leq t \leq 1} \ln ^{+}\left\|\Phi(\omega, t)^{ \pm}\right\| \in L^{1}(\Omega) .
$$

Then there exists an invariant subset $\widetilde{\Omega} \subset \Omega$ of full measure such that for each $\omega \in \widetilde{\Omega}$ the following statements hold:

(i) The limit $\lim _{|t| \rightarrow \infty}\left(\Phi(t, \omega)^{*} \Phi(t, \omega)\right)^{1 / 2 t}=: \Psi(\omega)$ exists.

(ii) Let $e^{\gamma_{1}(\omega)}<\cdots<e^{\gamma_{p(\omega)}(\omega)}$ be the different eigenvalues of $\Psi(\omega)$ with the multiplicities $l_{i}(\omega)$. Then we have that $p\left(\theta_{t} \omega\right)=p(\omega)$, $l_{i}\left(\theta_{t} \omega\right)=l_{i}(\omega)$, and $\gamma_{i}\left(\theta_{t} \omega\right)=\gamma_{i}(\omega)$ for $i=1, \ldots, p(\omega)$ and any $t \in \mathbb{R}$.

(iii) The functions $\omega \rightarrow p(\omega), \omega \rightarrow l_{i}(\omega)$ and $\omega \rightarrow \gamma_{i}(\omega)$ are measurable. In addition, if $\left(\Omega, \mathcal{F}, \mathbb{P}, \theta^{n}\right)$ is ergodic, then the functions $p(\omega)$, $l_{i}(\omega)$, and $\gamma_{i}(\omega)$ are invariant constants.

(iv) There exists a measurable map

$$
P: \Omega \rightarrow G l(d, \mathbb{C})
$$

satisfying $\|P(\omega)\|$ and $\left\|P^{-1}(\omega)\right\|$ are both tempered such that $\Phi(t, \omega)$ is conjugated to a block diagonal random dynamical system $\Psi(t, \omega)$ by $P(\omega)$ :

$$
P\left(\theta_{t} \omega\right) \Phi(t, \omega) P^{-1}(\omega)=\Psi(t, \omega),
$$

where $\Psi(t, \omega)=\operatorname{diag}\left(\Psi_{1}(t, \omega), \ldots, \Psi_{p}(t, \omega)\right)$ and $\Psi_{i}(t, \omega)$ are cocycles of size $l_{i}(\omega) \times l_{i}(\omega)$ with the same Lyapunov exponents.

Finally, we recall some of the hyperbolic properties of a non-autonomous system. By studying the exponential dichotomy we can characterize the asymptotic speed of the solutions tending to infinity, which implies a close relationship with the unique bounded solution of a non-autonomous system.

Lemma 7. Let $A$ be a $d \times d$ matrix in the Jordan normal form. Consider the following differential equation

$$
\dot{x}=(A+B(t)) x,
$$


where $B(t)$ is almost periodic in $t$. If

$$
\beta=\sup _{t \in \mathbb{R}}\|B(t)\| \leq \alpha /\left(36 K^{5}\right),
$$

where $\alpha=\mu / 4, \mu=\min \left\{\left|\operatorname{Re}\left(\lambda_{i}-\lambda_{j}\right)\right| \mid \operatorname{Re} \lambda_{i} \neq \operatorname{Re} \lambda_{j}\right\}$ and $K$ is a constant dependent on the dimension $d$, then there exists an almost periodic change of variables $x=y+H(t) y$ turning system (7) into

$$
\dot{y}=(A+\widehat{B}(t)) y \text {. }
$$

Here $m(\widehat{B})$ and $m(H) \subseteq m(B),\|H\| \leq 18 \alpha^{-1} K \beta,\|\widehat{B}\| \leq 3 K \beta$ and $\widehat{B}(t)$ is in the block diagonal form with respect to the different real parts of the eigenvalues of $A$.

As usual, $m(f)$ denotes the module of an almost periodic function $f$. The above toughness lemma comes from the classical research of the exponential dichotomy. A detailed proof can be found in [6]. Moreover, it is well known that the exponential dichotomy implies the existence of a unique bounded solution, which is almost periodic for almost periodic systems. Here, the statement of the following lemma is in $[\mathbf{6}]$, see also $[\mathbf{9}$, 10].

Lemma 8. For the non-homogeneous equation

$$
\dot{x}=A(t) x+f(t),
$$

where $A(t)$ and $f(t)$ are almost periodic functions, if the corresponding homogeneous equation $\dot{x}=A(t) x$ has an exponential dichotomy on $\mathbb{R}$, then there exits a unique almost periodic solution $\psi$ of that non-homogeneous equation which satisfies $m(\psi) \subset m(A(t) x+f(t))$.

\section{Linearization criterion}

In this part we provide the analytic linearization criterion for non-autonomous systems with diagonal linear parts and sub-exponential unbounded nonlinearities. The methods are enlightened from $[\mathbf{1 3}, \mathbf{1 6}, \mathbf{1 7}]$. See $[\mathbf{1 4}]$ for a weak version.

Consider the following non-autonomous differential systems

$$
\frac{d x}{d t}=\Lambda x+f(t, x),
$$

where $\Lambda=\operatorname{diag}\left(\lambda_{1}, \ldots, \lambda_{d}\right), f(t, 0) \equiv 0, D_{x} f(t, 0) \equiv 0$ and $f$ is analytic at $x=0$ for a fixed $t$. Consider $f=\sum_{l=1}^{d} \sum_{|k| \geq 2} f_{k}^{l}(t) x^{k} e_{l}$. For simplicity of the proof, we only consider that for all the monomials $f_{k}^{l}(t) x^{k} e_{l}$ satisfying $f_{k}^{l}(t) \not \equiv 0$ there exists a $\delta>0$ such that $\langle k, \lambda\rangle-\lambda_{l} \leq-|k| \delta$. The arguments for the other case are similar. 
When system (8) can be linearized, then there exist coordinates substitutions $x=y+h(t, y)$, which change the original system into $\dot{y}=\Lambda y$. By simple computation, it yields

$$
\partial_{t} h(t, y)+D_{y} h(t, y) \Lambda y-\Lambda h(t, y)=f(t, y+h(t, y)) .
$$

Denote two operators by $\operatorname{ad}_{\Lambda}: h(t, y) \mapsto \partial_{t} h(t, y)+D_{y} h(t, y) \Lambda y-\Lambda h(t, y)$ and $\mathrm{S}_{f}: h(t, y) \mapsto f(t, y+h(t, y))$. Then system (9) can be rewritten as

$$
\operatorname{ad}_{\Lambda} h(t, y)=\mathrm{S}_{f} h(t, y) .
$$

Therefore, the key is to detect the solution as the fixed point of above equation in the convenient functional space. Let us begin with the estimation of unbounded exponential nonlinearities.

Lemma 9. Let $\rho(t)$ and $M(t)$ be two positive continuous functions. Denote the positive function $\eta(t)$ by

$$
\eta(t)=\min \{1 /(C(d) M(t)), \rho(t) / 2,1\},
$$

where $C(d)=d \sum_{|k| \geq 0}(1 / 2)^{|k|}$. Assume that $f(t, x)$ is analytic in the domain $\{(t, x)|| x \mid \leq \rho(t)\}$ for a fixed $t, f(t, 0) \equiv 0, D_{x} f(t, 0) \equiv 0$ and

$$
\max _{|x| \leq \rho(t)}|f(t, x)| \leq M(t), \quad t \in \mathbb{R} .
$$

Then any positive function $\kappa(t)$ satisfying $\kappa(t) \leq \eta(t)$ can fulfill the condition

$$
\max _{|x| \leq \kappa(t)}|f(t, x)| \leq \kappa(t), \quad t \in \mathbb{R} .
$$

Moreover, the functions $\rho(t)$ and $M(t)$ satisfy

$$
\lim _{|t| \rightarrow \infty} \frac{1}{|t|} \ln ^{+} M(t)=0, \quad \lim _{|t| \rightarrow \infty} \frac{1}{|t|} \ln ^{-} \rho(t)=0 .
$$

Hence, the function $\eta$ satisfies $\lim _{|t| \rightarrow \infty} \frac{1}{t} \ln \eta(t)=0$.

Proof: By Cauchy estimation, we have that $\sup _{l}\left|f_{k}^{l}(t)\right| \leq M(t) / \rho^{|k|}(t)$. Therefore, for every fixed $t$, the conditions $f(t, 0) \equiv 0$ and $D_{x} f(t, 0) \equiv 0$ naturally imply that

$$
\begin{aligned}
|f(t, x)| & \leq \sum_{l=1}^{d} \sum_{|k| \geq 2}\left|f_{k}^{l}(t)\right||x|^{k} \\
& \leq|x|^{2} \sum_{l=1}^{d} \sum_{|k| \geq 0} \frac{M(t)}{\rho^{|k|}(t)}|x|^{|k|} \leq C(d) M(t)|x|^{2},
\end{aligned}
$$


where $C(d)=d \sum_{|k|>0}(1 / 2)^{|k|}$ for $|x| \leq \rho(t) / 2$. Then by formula (11) we know that $\lim _{|t| \rightarrow \infty} \frac{1}{|t|} \ln \eta(t)=0$ and $\kappa(t)$ admits (12). This completes the proof.

Now we introduce the modified majorant norms, which are defined in the domain $\{(t, x)|| x \mid \leq r \rho(t)\}$ by

$$
\llbracket f \rrbracket_{r, \rho}:=\sum_{|k|=1}^{\infty} \sum_{l=1}^{d} \sup _{t \in \mathbb{R}}\left\{\left|f_{k}^{l}(t)\right| \rho(t)^{|k|-1}\right\} r^{|k|}<\infty
$$

and

$$
\|f\|_{r, \rho}:=\sum_{|k|=1}^{\infty} \sum_{l=1}^{d} \sup _{t \in \mathbb{R}}\left\{\left|f_{k}^{l}(t)\right| \rho(t)^{|k|}\right\} r^{|k|}<\infty
$$

for any formal series $f(t, x)=\sum_{|k|=1}^{\infty} \sum_{l=1}^{d} f_{k}^{l}(t) x^{k} e_{l}$. One will easily see that they are natural generations for the definition of majorant norm because for any fixed $t \in \mathbb{R}$ we have that

$$
\sum_{|k|=1}^{\infty} \sum_{l=1}^{d}\left|f_{k}^{l}(t)\right| \rho(t)^{|k|} r^{|k|}=\mathcal{M} f(t, r \rho(t), \ldots, r \rho(t))=\mathcal{M} f(t, r \rho(t) \widehat{e}),
$$

associated with the classical majorant operator

$$
\mathcal{M}: \sum_{\alpha \in \mathbb{Z}_{d}^{+}} c_{\alpha} x^{\alpha} \mapsto \sum_{\alpha \in \mathbb{Z}_{d}^{+}}\left|c_{\alpha}\right| x^{\alpha}
$$

where $\widehat{e}=(1, \ldots, 1) \in \mathbb{C}^{d}$. Especially, notice that the above definitions, when $f$ is independent of the variable $t$, agree with the classical ones in [13]. That is, $\llbracket f \rrbracket_{r, 1}=\|f\|_{r, 1}=\llbracket f \rrbracket_{r}$.

Consider $B_{r}=\left\{h \mid h(t, 0)=0, \square h \rrbracket_{r, \kappa} \leq r, h \in \mathcal{C}_{k \delta}(\Lambda)\right\}$. Then we study the operators $\operatorname{ad}_{\Lambda}$ and $\mathrm{S}_{f}$ in $B_{r}$. The key is to show that $\operatorname{ad}_{\Lambda}$ has a bounded reverse and $\mathrm{S}_{f}$ is strongly contracting in some convenient functional space.

Lemma 10. The operator $\operatorname{ad}_{\Lambda}$ has a bounded inverse in the space $\left(B_{r},\left[\cdot \square_{r, \kappa}\right)\right.$, provided that there exists a positive constant $\tau<\delta$ such that

$$
\frac{\kappa(t+h)}{\kappa(t)} \leq e^{\tau|h|}, \quad t, h \in \mathbb{R}
$$


Proof: $\operatorname{Let}^{\operatorname{ad}_{\Lambda}} g=f$ with $\llbracket f \rrbracket_{r, \kappa}<\infty$. Then for the expansion $f=$ $\sum f_{k}^{l}(t) x^{k} e_{l}$, the solution $g=\sum g_{k}^{l}(t) x^{k} e_{l}$, which follows from the expression

$$
g_{k}^{l}(t)= \begin{cases}-\int_{t}^{\infty} e^{(s-t)\left(\langle k, \lambda\rangle-\lambda_{l}\right)} f_{k}^{l}(s) d s, & \langle k, l\rangle<\lambda_{l} \\ \int_{-\infty}^{t} e^{(s-t)\left(\langle k, \lambda\rangle-\lambda_{l}\right)} f_{k}^{l}(s) d s, & \langle k, l\rangle>\lambda_{l}\end{cases}
$$

is unique in the sense of the Lyapunov exponent less than $\delta$.

Then consider $c_{k}^{l}(g)=\sup _{t \in \mathbb{R}}\left|g_{k}^{l}(t)\right| \kappa(t)^{|k|-1}$. By formula (14) we obtain

$$
\begin{aligned}
\left|g_{k}^{l}(u)\right| \kappa(u)^{|k|-1} & \leq \int_{u}^{\infty} e^{(s-u)\left(\langle k, \lambda\rangle-\lambda_{l}\right)}\left|f_{k}^{l}(s)\right| \kappa(u)^{|k|-1} d s \\
& =\int_{0}^{\infty} e^{t\left(\langle k, \lambda\rangle-\lambda_{l}\right)}\left|f_{k}^{l}(u+t)\right| \kappa(u+t)^{|k|-1}(\kappa(u) / \kappa(u+t))^{|k|-1} d t \\
& \leq c_{k}^{l}(f) \int_{0}^{\infty} e^{-\delta|k| t} e^{\tau(|k|-1) t} d t \leq \frac{c_{k}^{l}(f)}{\delta-\tau},
\end{aligned}
$$

which implies $\llbracket g \rrbracket_{r, \kappa} \leq \llbracket f \rrbracket_{r, \kappa} /(\delta-\tau)$ for every positive $r$. This completes the proof.

Next three lemmas confirm the strongly contraction of $\mathrm{S}_{f}$.

Lemma 11. If $f$ and $h \in \mathcal{C}_{k \delta}(\Lambda)$, then we have that $\mathrm{S}_{f}(h) \in \mathcal{C}_{k \delta}(\Lambda)$.

Proof: Without loss of generality, we denote the coefficients simply by $\sim$, whatever depending on $t$ or not. So $f=\sum \sim x^{k} e_{l}$ for $\langle k, \lambda\rangle-\lambda_{l} \leq-|k| \delta$.

We have that

$$
\begin{aligned}
\mathrm{S}_{f}(h) & =\sum f_{k}^{l}(x+h(\sim, x))^{k} e_{l} \\
& =\sum \sim \prod_{s=1}^{d}\left(\sim x_{s}+\sum_{t} \sim x^{m_{s t}}\right)^{k_{s}} e_{l} \\
& =\sum \sim\left(\prod_{s=1}^{d} x_{s}^{i_{s 0}}\right) x^{\sum_{s=1}^{d} \sum_{t \geq 1} i_{s t} m_{s t}} e_{l} .
\end{aligned}
$$


Here $k=\left(k_{1}, \ldots, k_{d}\right), \sim x^{m_{s t}} e_{s}$ is a monomial of $h$ with the non-zero coefficient, and $\sum_{t=0}^{\infty} i_{s t}=k_{s}$ for $s=1, \ldots, d$. It then leads to

$$
\begin{aligned}
& -\lambda_{l}+\sum_{s=1}^{d} i_{s 0} \lambda_{s}+\sum_{s=1}^{d} \sum_{t \geq 1}\left\langle i_{s t} m_{s t}, \lambda\right\rangle \\
\leq & -\lambda_{l}+\sum_{s=1}^{d} i_{s 0} \lambda_{s}+\sum_{s=1}^{d} \sum_{t \geq 1} i_{s t}\left(-\delta\left|m_{s t}\right|+\lambda_{s}\right) \\
= & -\lambda_{l}+\langle k, \lambda\rangle-\delta \sum_{s=1}^{d} \sum_{t \geq 1} i_{s t}\left|m_{s t}\right| \\
\leq & -\delta\left(|k|+\sum_{s=1}^{d} \sum_{t \geq 1} i_{s t}\left|m_{s t}\right|\right) \\
\leq & -\delta\left(\sum_{s=1}^{d} i_{s 0}+\sum_{s=1}^{d} \sum_{t \geq 1} i_{s t}\left|m_{s t}\right|\right) .
\end{aligned}
$$

That is, we get $\mathrm{S}_{f}(h) \in \mathcal{C}_{k \delta}(\Lambda)$, which completes the proof.

Lemma 12. Assume that $f(t, 0)=g(t, 0)=0$. Then the following statements hold.

(i) $\square f g \rrbracket_{r, \kappa} \leq\|f\|_{r, \kappa} \llbracket g \rrbracket_{r, \kappa}$, provided that both $\|f\|_{r, \kappa}$ and $\square g \square_{r, \kappa}$ are finite.

(ii) $\|f \circ g\|_{r, \kappa} \leq\|f\|_{\tau, \kappa}$, provided that $\tau=\llbracket g \rrbracket_{r, \kappa}$.

Proof: For a fixed $t$, we have that

$$
\sum_{|k|=1}^{\infty} \sum_{l=1}^{d}\left|f_{k}^{l}(t)\right| \kappa^{|k|-1}(t) r^{|k|}=\kappa^{-1}(t) \mathcal{M} f(t, r \kappa(t) \widehat{e}) .
$$

Consequently, by Proposition 5, it implies that

$$
\begin{aligned}
\kappa^{-1}(t) \mathcal{M}(f g)(t, r \kappa(t) \widehat{e}) & \leq \kappa^{-1}(t) \mathcal{M} f(t, r \kappa(t) \widehat{e}) \mathcal{M} g(t, r \kappa(t) \widehat{e}) \\
& \leq \mathcal{M} f(t, r \kappa(t) \widehat{e}) \kappa^{-1}(t) \mathcal{M} g(t, r \kappa(t) \widehat{e})
\end{aligned}
$$

Thus $\llbracket f g \rrbracket_{r, \kappa} \leq\|f\|_{r, \kappa} \rrbracket g \rrbracket_{r, \kappa}$, which proves (i). The proof of (ii) follows similarly from the fact that

$$
\begin{aligned}
\mathcal{M}(f \circ g)(t, r \kappa(t) \widehat{e}) & \leq \mathcal{M} f(t, \mathcal{M} g(t, r \kappa(t) \widehat{e}) \widehat{e}) \\
& \leq \mathcal{M} f\left(t, \kappa(t) \kappa^{-1}(t) \mathcal{M} g(t, r \kappa(t) \widehat{e}) \widehat{e}\right) \\
& \leq \mathcal{M} f(t, \tau \kappa(t) \widehat{e}) .
\end{aligned}
$$

This completes the proof. 
Here the operator $\mathrm{S}_{f}$ is called strongly contracting in the ball $\left(B_{r}, \square \cdot \square_{r, \kappa}\right)$, provided that the following conditions are fulfilled

(i) $\mathrm{S}_{f}\left(B_{r}\right) \subseteq B_{r}$,

(ii) $\square \mathrm{S}_{f} h-\mathrm{S}_{f} \widetilde{h} \rrbracket_{r, \kappa} \leq C(r) \llbracket h-\widetilde{h} \rrbracket_{r, \kappa}$, where $C(r) \rightarrow 0$ as $r \rightarrow 0$.

Lemma 13. Assume that $f(t, 0)=0, D_{x} f(t, 0)=0$ and

$$
\max _{|x| \leq \kappa(t)}|f(t, x)| \leq \kappa(t), \quad t \in \mathbb{R} .
$$

Then the operator $\mathrm{S}_{f}$ is strongly contracting in the ball $\left(B_{r},\left[\cdot \square_{r, \kappa}\right)\right.$ for $r$ small enough.

Proof: By Lemma 11, it is proved that $\mathrm{S}_{f}\left(\mathcal{C}_{k \delta}(\Lambda)\right) \subseteq \mathcal{C}_{k \delta}(\Lambda)$. Then notice that

$$
\mathrm{S}_{f} h(t, x)=\int_{0}^{1} D_{x} f(t, u(x+h(t, x))(x+h(t, x)) d u
$$

and

$$
g(t, x)=\int_{0}^{1} D_{x} f(t, x+u h(t, x)+(1-u) \widetilde{h}(t, x))(h-\widetilde{h})(t, x) d u,
$$

where $g=\mathrm{S}_{f} h-\mathrm{S}_{f} \tilde{h}$. Additionally $\llbracket x \rrbracket_{r, \kappa}=r$. Thus by Lemma 12 we obtain that

$\square \mathrm{S}_{f} h \rrbracket_{r, \kappa} \leq\left\|D_{x} f\right\|_{\tau, \kappa}\left(\llbracket h \rrbracket_{r, \kappa}+\llbracket x \rrbracket_{r, \kappa}\right), \quad \llbracket g \rrbracket_{r, \kappa} \leq\left\|D_{x} f\right\|_{\tau, \kappa} \rrbracket h-\widetilde{h} \rrbracket_{r, \kappa}$,

where $\tau \leq \llbracket x \rrbracket_{r, \kappa}+\max \left\{\llbracket h \rrbracket_{r, \kappa}, \llbracket \widetilde{h} \rrbracket_{r, \kappa}\right\} \leq 3 r$.

Now we estimate the upper bound of $\left\|D_{x} f\right\|_{3 r, \kappa}$. By Lemma 12 and the Cauchy estimation we obtain that $\left|f_{k}^{l}(t)\right| \leq \kappa^{1-|k|}(t)$ for a fixed $t$. Thus, when $r<1 / 6$, we have that

$$
\begin{aligned}
\left\|D_{x} f\right\|_{3 r, \kappa} & \leq d \sum_{|k|=2}^{\infty}|k|\left|f_{k}^{l}(t)\right| \kappa^{|k|-1}(t)(3 r)^{|k|-1} \\
& \leq d \sum_{|k|=2}^{\infty}|k|(3 r)^{|k|-1} \leq d r \sum_{|k|=0}^{\infty}(|k|+2) / 2^{|k|} .
\end{aligned}
$$

This completes the proof.

Finally, in this context we restate Theorem 1 as follows and give the proof out. 
Theorem 14. Let $\rho(t)$ and $M(t)$ be two positive continuous functions. Assume that in system (8) the function $f(t, x) \in \mathcal{C}_{k d}(\Lambda)$ is analytic in the domain $\{(t, x)|| x \mid \leq \rho(t)\}$ for a fixed $t, f(t, 0)=0, D_{x} f(t, 0)=0$ and

$$
\max _{|x| \leq \rho(t)}|f(t, x)| \leq M(t), \quad t \in \mathbb{R} .
$$

If there exits a positive function $\kappa(t)$ satisfying conditions (11) and (13), then there exist coordinates substitutions $x=y+h(y, t)$, which are analytic in the domain $\left\{(t, x)|| x \mid \leq r_{0} \kappa(t)\right\}$ for a fixed $t$ and some $r_{0}>0$, changing system (8) into its linear part. Moreover, if functions $\rho(t)$ and $M(t)$ additionally satisfy

$$
\lim _{|t| \rightarrow \infty} \frac{1}{|t|} \ln ^{+} M(t)=0, \quad \lim _{|t| \rightarrow \infty} \frac{1}{|t|} \ln ^{-} \rho(t)=0,
$$

then, for any fixed $y$, the function $h(t, y)$ admits

$$
\lim _{|t| \rightarrow \infty} \frac{1}{|t|} \ln |h(t, y)|=0 .
$$

Proof: The key is to prove that equation (10) has an analytic solution in $\left(B_{r},[] \cdot \square_{r, \kappa}\right)$ via the Contraction Mapping Principle for some convenient $\kappa(t)$.

Consider the composition operator $\operatorname{ad}_{\Lambda}^{-1} \circ \mathrm{S}_{f}$ in the space $\left(B_{r}, \square \cdot \square_{r, \kappa}\right)$, where both $\operatorname{ad}_{\Lambda}$ and $\mathrm{S}_{f}$ are given in equation (10). On one hand, the operator $\operatorname{ad}_{\Lambda}^{-1}$ is bounded by Lemma 10, whose norm is independent of $r$. On the other hand, the shift operator $\mathrm{S}_{f}$ is strongly contracting with the Lipschitz constant going to zero with $r \rightarrow 0$ as $O(r)$. Thus the composition will be contracting in $\left(B_{r},\left[\cdot \square_{r, \kappa}\right)\right.$ with the rate $O(1) \cdot O(r)=O(r)$ as $r \rightarrow 0$. By the Contracting Mapping Principle, we can choose $r$ small enough such that there exists a unique fixed point of the equation

$$
h=\left(\operatorname{ad}_{\Lambda}^{-1} \circ \mathrm{S}_{f}\right) h \text { for } h \in\left(B_{r},\left[\cdot \square_{r, \kappa}\right) .\right.
$$

Thus, equation (10), or the equivalent system of the form (9), has an analytic solution in the domain $\{(t, x)|| x \mid<r \kappa(t)\}$, which linearizes system (8). This completes the proof.

\section{Autonomous systems}

In this part we study autonomous differential systems in order to extend the results in [3] for the non-diagonal case. Consider the following analytic system

$$
\dot{x}=A x+f(x),
$$


where $A=D+N$ is in the Jordan normal form with the diagonal and nilpotent parts $D$ and $N$, respectively. If system (15) can be changed into $\dot{y}=A y$ by analytic coordinates substitutions $x=y+h(y)$, the traditional way is to study the equality

$$
D_{y} h A y-A h(y)=f(y+h(y)) \text {. }
$$

In general it is impossible to seek the explicit expression of the operator $\operatorname{ad}_{A}: h \mapsto D_{y} h A y-A h(y)$ except when $A$ is diagonal. Consequently, we use another way to study it. Doing non-autonomous coordinates substitutions $x=e^{N t} u$, system (15) can be changed into the non-autonomous one

$$
\dot{u}=D u+e^{-N t} f\left(e^{N t} u\right) .
$$

Then we can apply Theorem 1 . So it is necessary to analyze the conditions of the linearization criterion one by one.

First of all, we verify that system (16) formally satisfies the conditions of Theorem 1 . Let $E$ be the classical identity matrix.

Lemma 15. Assume that $A=D+N$ is in the Jordan normal form with the diagonal $D$ and nilpotent $N$. Then $e^{-N t} f\left(e^{N t} u\right) \in \mathcal{C}_{k \delta}(D)$, provided that $f \in \mathcal{C}_{k \delta}(D)$.

Proof: Consider $B=e^{N t}$ or $e^{-N t}$. Notice that $B$ has the expansion of the form $E \pm N t+\cdots+( \pm N t)^{d} / d$ !. Since in the rest proof the coefficients are not important, we shall not make the distinction between their signs. Moreover, if $N^{m} \neq 0$, then any non-zero $(i, j)$-th entry implies $\lambda_{i}=\lambda_{j}$, hence $B f(x)$ has a monomial $x^{k} e_{i}$ and $\langle k, \lambda\rangle-\lambda_{i}=\langle k, \lambda\rangle-\lambda_{j}$. Thus we have that $B f(x) \in \mathcal{C}_{k \delta}(D)$. Then by straightforward computation it is easy to get $B x=\sum\left(\lambda_{i} x_{i}+\sim x_{i+1}+\cdots+\sim x_{i+m_{i}}\right) e_{i}$, where $\lambda_{i}=$ $\cdots=\lambda_{i+m_{i}}$. So a typical monomial of $f(B x)$ takes the form $\prod\left(\lambda_{i} x_{i}+\sim\right.$ $\left.x_{i+1}+\cdots+\sim x_{i+k_{i}}\right)^{k_{i}} e_{l}$, whose monomials preserve $\langle k, \lambda\rangle-\lambda_{l}$ invariant. So we get $f(B x) \in \mathcal{C}_{k \delta}(D)$ and the proof is finished.

Let $|\cdot|_{0}$ be the operator norm of the matrix given by

$$
|A|_{0}:=\sup _{\|x\|=1}\|A x\|=\sup _{\|x\|=1} \sqrt{x^{*} A^{*} A x}
$$

where ${ }^{*}$ is the classical notation for the conjugate transpose matrix. Then we obtain the function $\kappa(t)$, which is key to determine the conjugated domain. 
Lemma 16. Assume $A=D+N$ is in the Jordan normal form with the diagonal $D$ and non-zero nilpotent $N$. Let $\rho_{1}(t)=\left|e^{N t}\right|_{0}$ and $\rho_{2}(t)=$ $\left|e^{-N t}\right|_{0}$. Then for every positive $\sigma$ there exits a function $\kappa(t)$ such that

(i) $\kappa(s) / \kappa(t) \leq e^{\sigma|s-t|}$ for $s, t \in \mathbb{R}$.

(ii) $C_{2} \kappa(t) \leq \rho_{i}(t) \leq C_{1} \kappa(t)$ for $i=1,2$ and positive constants $C_{1}$ and $C_{2}$.

Proof: Consider $s$ satisfying $N^{s} \neq 0$ and $N^{s+1}=0$. Since we have that $e^{ \pm N t}=E \pm N t+\cdots+( \pm 1)^{s} N^{s} t^{s} / s !$, we obtain that

$$
\rho_{i}(t) \sim o\left(|t|^{s}\right), \quad|t| \rightarrow \infty .
$$

So $C_{2}\left(1+|t|^{s}\right) \leq \rho_{i}(t) \leq C_{1}\left(1+|t|^{s}\right)$ for $i=1$ and 2. Let $g(t)=1+|t|^{s}$. Then the function $g(t): \mathbb{R} \rightarrow \mathbb{R}^{+}$is of class $C^{1}$ and satisfies

$$
\lim _{|t| \rightarrow \infty} \frac{g^{\prime}(t)}{g(t)}=\lim _{|t| \rightarrow \infty}\left|(\ln g(t))^{\prime}\right|=0, \quad \lim _{|t| \rightarrow \infty} g(t)=\infty .
$$

Thus for that $\sigma$ there exits $N$ such that for $|t| \geq N$ and we have that $\left|(\ln g(t))^{\prime}\right| \leq \sigma$. Let $\bar{b}=\max _{[-N, N]} g(t)$. Remark $a_{1} \in[-\infty, N]$ and $a_{2} \in[N, \infty]$ such that $\rho\left(a_{1}\right)=\rho\left(a_{2}\right)=\bar{b}$. Therefore, we can define

$$
\kappa(t)= \begin{cases}\bar{b}, & t \in\left[a_{1}, a_{2}\right] ; \\ g(t), & t \in \mathbb{R} \backslash\left[a_{1}, a_{2}\right],\end{cases}
$$

satisfying $g(t) \leq \kappa(t)$. Moreover, we also have that

$$
\left|\int_{t}^{s} \frac{\kappa^{\prime}(u)}{\kappa(u)} d u\right|=|\ln \kappa(s)-\ln \kappa(t)| \leq \sigma|s-t|,
$$

which implies (i). Finally $\bar{b}=\min _{[-N, N]} g(t)$, then $(\bar{b} / \underline{b}) g(t) \geq \kappa(t)$, which proves the statement (ii).

Next lemma guarantees the preserving of autonomous structures.

Lemma 17. Assume that $f \in \mathcal{C}_{k \delta}(D)$ and that equation (16) can be linearized, then the transformation has the form $u=x+e^{-N t} h\left(e^{N t} x\right)$ in the sense of formal series with respect to $h$.

Proof: Consider $\widetilde{f}(t, x)=e^{-N t} f\left(e^{N t} x\right)$. If $\widetilde{h}(t, x)=e^{-N t} h\left(e^{N t} x\right)$, then we obtain

$$
\begin{aligned}
\mathrm{S}_{\widetilde{f}}(\widetilde{h}) & =e^{-N t} f\left(e^{N t}\left(x+e^{-N t} h\left(e^{N t} x\right)\right)\right) \\
& =e^{-N t} f\left(e^{N t} x+h\left(e^{N t} x\right)\right) \\
& =e^{-N t} F\left(e^{N t} x\right)
\end{aligned}
$$


where $F(x)=f(x+h(x))$. Next, by formula (14) for a fixed $k$ we have that

$$
\operatorname{ad}_{D}^{-1} \tilde{f}_{k}(t, x)= \begin{cases}-\int_{t}^{\infty} e^{(s-t)\left(\langle k, \lambda\rangle E_{d}-D\right)} e^{-N s} f_{k}\left(e^{N s} x\right) d s, & \langle k, l\rangle<\lambda_{l} \\ \int_{-\infty}^{t} e^{(s-t)\left(\langle k, \lambda\rangle E_{d}-D\right)} e^{-N s} f_{k}\left(e^{N s} x\right) d s, & \langle k, l\rangle>\lambda_{l},\end{cases}
$$

where $\tilde{f}_{k}(t, x)=\sum_{l=1}^{d} \widetilde{f}_{k}^{l}(t) x^{k} e_{l}$. For simplicity, we only consider the case $\langle k, l\rangle<\lambda_{l}$ for all $l$. The other case follows with similar arguments. Let $\widetilde{g}_{k}(t, x)=\operatorname{ad}_{D}^{-1} \widetilde{f}_{k}(t, x)$, then we notice that

$$
\begin{aligned}
e^{N t} \widetilde{g}_{k}\left(t, e^{-N t} x\right) & =-\int_{t}^{\infty} e^{(s-t)\left(\langle k, \lambda\rangle E_{d}-D\right)} e^{-N(s-t)} f_{k}\left(e^{N(s-t)} x\right) d s \\
& =-\int_{0}^{\infty} e^{u\left(\langle k, \lambda\rangle E_{d}-D\right)} e^{-N u} f_{k}\left(e^{N u} x\right) d u=G(x)
\end{aligned}
$$

is independent of the variable $t$. So $\widetilde{g}_{k}(t, x)=e^{-N t} G\left(e^{N t} x\right)$. Then the proof follows by an induction procedure.

Here we specially mentioned again that for an analytic function $h(x)$, independent of the time variable $t$, the classical definition can be made by

$$
\square f \rrbracket_{r}=\sum_{|k|=1}^{\infty} \sum_{l=1}^{d}\left|f_{k}^{l}\right| r^{|k|}=\mathcal{M} f(r, \ldots, r) .
$$

Now we restate Theorem 2 as follows and give the proof out.

Theorem 18. Assume that $f(x)$ is analytic at the origin, $f(x)=O\left(\|x\|^{2}\right)$ as $x \rightarrow 0, A$ is in the Jordan normal form and $f \in \mathcal{C}_{k d}(D)$ in system (15). Then system (15) can be analytically linearized.

Proof: The case $N=0$, i.e. $A$ in the diagonal form, follows by [3].

If $N \neq 0$, the transformation $x=e^{N t} u$ changes system (15) to system (16). Let $\tilde{f}(t, u)=e^{-N t} f\left(e^{N t} u\right)$. Since $f$ is analytic at the origin, we can assume that $\max _{|x| \leq r_{1}}|f(x)| \leq M_{1}$, which implies

$$
\max _{|x| \leq r / \rho_{1}(t)}|\tilde{f}(t, u)| \leq M_{1} \rho_{2}(t), \quad t \in \mathbb{R},
$$

where $\rho_{1}$ and $\rho_{2}$ are given in Lemma 16. Notice that by Lemma 16 again, choosing $\sigma=\delta / 2$ and

$$
C=\min \left\{\frac{1}{C(d) M_{1} C_{1}}, \frac{r}{2 C_{1}}\right\}
$$

$\widetilde{\kappa}(t)=C \kappa(t)^{-1}$ satisfies the conditions (11) and (13). In addition, by Lemma 15 and Theorem 14 we obtain coordinates transformations $u \mapsto$ 
$u+\widetilde{h}(t, u)$ with $\llbracket \widetilde{h} \rrbracket_{r_{0}, \widetilde{\kappa}(t)} \leq r_{0}$ for some $r_{0}$ linearizing system (16). Finally, by Lemma 17 we obtain $\widetilde{h}=e^{-N t} h\left(e^{N t} u\right)$. Consequently, we have that $\widetilde{h}(0, u)=h(u)$ and

$$
r_{0} \geq \llbracket \widetilde{h} \rrbracket_{r_{0}, \widetilde{\kappa}(t)} \geq \sum_{|k|=1}^{\infty} \sum_{l=1}^{d}\left|f_{k}^{l}(0)\right| \rho(0)^{|k|-1} r_{0}^{|k|}=\frac{\llbracket h \rrbracket_{r_{0} \rho(0)}}{\rho(0)}
$$

Then the analytic transformation $y=x+h(x)$ linearizes system (15) and the proof is complete.

\section{Random dynamical systems}

In this part we consider the random system associated with the dynamical system $\left(\Omega, \mathcal{F}, \mathbb{P},\left(\theta_{t}\right)_{t \in \mathbb{R}}\right)$

$$
\dot{x}_{t}=A\left(\theta_{t} \omega\right) x_{t}+f\left(\theta_{t} \omega, x\right)=F\left(\theta_{t} \omega, x\right),
$$

under the following hypotheses:

(i) The condition of Multiplicative Ergodic Theorem is satisfied, i.e.

$$
\alpha^{ \pm}(\omega):=\sup _{0 \leq t \leq 1} \ln ^{+}\left\|\Phi(\omega, t)^{ \pm}\right\| \in L^{1} .
$$

(ii) The function $F\left(\theta_{t} \omega, x\right)$ is analytic in $x$ for a fixed $t$ and there exist $\rho(\omega)$ tempered from below and $M(\omega)$ tempered from above such that

$$
\max _{|x| \leq \rho\left(\theta_{t} \omega\right)}\left|f\left(\theta_{t} \omega, x\right)\right|=M\left(\theta_{t} \omega\right)<\infty .
$$

(iii) $F\left(\theta_{t} \omega, x\right), \rho\left(\theta_{t} \omega\right)$ and $M\left(\theta_{t} \omega\right)$ are all continuous in $t$ for $\omega \mathbb{P}$ a.e. Applying our methods, we give a new proof of Poincaré analytic linearization theorem for random dynamical systems. See [16] for another one by homotopy methods.

First we ensure that system (18) is regular $\mathbb{P}$ a.e.

Lemma 19. Consider the linear random system associated with the dynamical system $\left(\Omega, \mathcal{F}, \mathbb{P},\left(\theta_{t}\right)_{t \in \mathbb{R}}\right)$ as follows

$$
\dot{x}_{t}=A\left(\theta_{t} \omega\right) x_{t},
$$

satisfying hypotheses (i) and (iii). Denote the d-tuple positive reals $\gamma(\omega)=\left(\gamma_{1}(\omega), \ldots, \gamma_{d}(\omega)\right)$ by Lyapunov exponents without multiplicities. Then by the coordinates substitution $x_{t}=P(\omega, t) y_{t}$, system (19) can be changed into an invariant system like

$$
\dot{y}_{t}=\Lambda(\omega) y_{t}=\operatorname{diag}(\gamma(\omega)) y_{t}=\operatorname{diag}\left(\gamma\left(\theta_{t} \omega\right)\right) y_{t} .
$$

Moreover, the following statements hold: 
(i) $P(\omega, t)=P_{1}\left(\theta_{t} \omega\right) P_{2}(\omega, t)$, where $P_{1}(\omega)$ and $P_{1}^{-1}(\omega)$ are both tempered.

(ii) $P_{2}$ satisfies the cocycle property, i.e. $P_{2}(\omega, t+s)=P_{2}\left(\theta_{t} \omega, s\right) P_{2}(\omega, t)$ and $P_{2}(\omega, t) \Lambda(\omega)=\Lambda(\omega) P_{2}(\omega, t)$. And all Lyapunov exponents of $P_{2}(\omega, t)$ are zero.

We especially note that such transformations cannot admit random diffeomorphisms except that all the Lyapunov exponents are simple.

Proof: By Lemma 6(iv), i.e. Corollary 4.3.12 in [2], there exists a transformation $x_{t}=P_{1}\left(\theta_{t} \omega\right) y_{t}$ such that

$$
\widetilde{\Phi}(\omega, t)=P_{1}\left(\theta_{t} \omega\right) \Phi(\omega, t) P_{1}(\omega)^{-1},
$$

where $\widetilde{\Phi}(\omega, t)$ is a block diagonal one

$$
\widetilde{\Phi}(\omega, t)=\operatorname{diag}\left(\widetilde{\Phi}_{1}(\omega, t), \ldots, \widetilde{\Phi}_{q(\omega)}(\omega, t)\right)
$$

with the $l_{i}(\omega) \times l_{i}(\omega)$ block $\widetilde{\Phi}_{i}(\omega, t)$ admitting one point spectrum for $i=$ $1, \ldots, q(\omega)$. Moreover, the transfer matrix $P_{1}(\omega)$ and its inverse $P_{1}^{-1}(\omega)$ are both tempered. Then consider $\widehat{P}_{i}(\omega, t)=e^{-\gamma_{i}(\omega) t} \widetilde{\Phi}_{i}(\omega, t)$ and $\widehat{P}_{i}$ is a linear cocycle with all zero Lyapunov exponents. Let $P_{2}(\omega, t)=$ $\operatorname{diag}\left(\widehat{P}_{1}(\omega, t), \ldots, \widehat{P}_{l_{q(\omega)}}(\omega, t)\right)$. Then $P(\omega, t)=P_{1}\left(\theta_{t} \omega\right) P_{2}(\omega, t)$ makes system (19) transform into system (20), which proves (i). In addition, result (ii) follows from

$$
\begin{aligned}
\widehat{P}_{i}(\omega, t+s) & =e^{-\gamma(s+t)} \widetilde{\Phi}_{i}(\omega, s+t) \\
& =e^{-\gamma(s+t)} \widetilde{\Phi}_{i}\left(\theta_{t} \omega, s\right) \widetilde{\Phi}_{i}(\omega, t)=\widehat{P}_{i}\left(\theta_{t} \omega, s\right) \widehat{P}_{i}(\omega, t)
\end{aligned}
$$

and $\widehat{P}_{i}(\omega, t) \gamma_{i}(\omega) E_{l_{i}(\omega)}=\gamma_{i}(\omega) \widehat{P}_{i}(\omega, t)$.

Now, by Lemma 19, doing substitutions $x_{t}=P(\omega, t) y_{t}$ to system (18), we obtain

$$
\dot{y}_{t}=\Lambda(\omega) y_{t}+P^{-1}(\omega, t) f\left(\theta_{t} \omega, P(\omega, t) y_{t}\right) .
$$

Then we consider the corresponding random dynamical system adding an auxiliary parameter $s$

$$
\dot{y}_{t}=\Lambda(\omega) y_{t}+P^{-1}\left(\theta_{s} \omega, t\right) f\left(\theta_{t+s} \omega, P\left(\theta_{s} \omega, t\right) y_{t}\right) .
$$

Lemma 20. Under hypotheses (i), (ii) and (iii) if the Lyapunov exponents $\gamma(\omega)=\left(\gamma_{1}(\omega), \ldots, \gamma_{d}(\omega)\right)$ have the same signs and are nonresonant, then the linearized transformation of system (22) has the form $u_{t}=y_{t}+P^{-1}\left(\theta_{s} \omega, t\right) h\left(\theta_{t+s} \omega, P\left(\theta_{s} \omega, t\right) y_{t}\right)$ in the sense of formal series. 
Proof: System (18) can be formally linearized using [2] or [16]. So, without loss of generality, we can assume that the degree of $f$ with respect to the variable $u$ is greater than $\max \{\bar{\gamma}(\omega) / \gamma(\omega), \gamma(\omega) / \bar{\gamma}(\omega)\}$ in system (22), where $\bar{\gamma}(\omega)=\max _{i}\left\{\gamma_{i}(\omega)\right\}$ and $\gamma(\omega)=\min _{i}\left\{\gamma_{i}(\omega)\right\}$.

Then the proof follows similarly to the one of Lemma 17. Consider

$$
\widetilde{f}(s, \omega, t, y)=P^{-1}\left(\theta_{s} \omega, t\right) f\left(\theta_{t+s} \omega, P\left(\theta_{s} \omega, t\right) y\right) .
$$

If $\widetilde{h}(s, \omega, t, y)=P^{-1}\left(\theta_{s} \omega, t\right) h\left(\theta_{t+s} \omega, P\left(\theta_{s} \omega, t\right) y\right)$, then we obtain

$$
\begin{aligned}
\mathrm{S}_{\widetilde{f}}(\widetilde{h}) & =P^{-1}\left(\theta_{s} \omega, t\right) f\left(\theta_{t+s} \omega, P\left(\theta_{s} \omega, t\right) y+h\left(\theta_{t+s} \omega, P\left(\theta_{s} \omega, t\right) y\right)\right) \\
& =P^{-1}\left(\theta_{s} \omega, t\right) F\left(\theta_{t+s} \omega, P\left(\theta_{s} \omega, t\right) y\right),
\end{aligned}
$$

where $F(\cdot, y)=f(\cdot, y+h(\cdot, y))$.

Without loss of generality, we assume that all the Lyapunov exponents are positive. Let $\widetilde{f}_{k}(s, \omega, t, y)=\sum_{l=1}^{d} \widetilde{f}_{k}^{l}(s, \omega, t) y^{k} e_{l}$ and $\widetilde{g}_{k}(s, \omega, t, y)=$ $\operatorname{ad}_{\Lambda}^{-1} \widetilde{f}_{k}(s, \omega, t, y)$. Therefore, still by formula (14) for a fixed $k$ we have that

$$
\begin{gathered}
\widetilde{g}_{k}(s, \omega, t, y)=-\int_{t}^{\infty} e^{(a-t)\left(\langle k, \lambda\rangle E_{d}-\Lambda\right)} P^{-1}\left(\theta_{s} \omega, a\right) \widetilde{f}_{k}\left(\theta_{a+s} \omega, P\left(\theta_{s} \omega, a\right) y\right) d a \\
=-\int_{0}^{\infty} e^{u\left(\langle k, \lambda) E_{d}-\Lambda\right)} P^{-1}\left(\theta_{s} \omega, u+t\right) \\
\quad \times \widetilde{f}_{k}\left(\theta_{s+u+t} \omega, P\left(\theta_{s} \omega, u+t\right) y\right) d u .
\end{gathered}
$$

Since by Lemma 19(ii) we get that

$$
P\left(\theta_{s} \omega, u+t\right)=P_{1}\left(\theta_{s+t+u} \omega\right) P_{2}\left(\theta_{s} \omega, u+t\right)
$$

and $P_{2}(\omega, t) \Lambda(\omega)=\Lambda(\omega) P_{2}(\omega, t)$. Then by setting

$$
\begin{aligned}
G(\omega, x)=-P_{1}(\omega) \int_{0}^{\infty} & e^{u\left(\langle k, \lambda\rangle E_{d}-\Lambda\right)} P_{2}^{-1}(\omega, u) P_{1}^{-1}\left(\theta_{u} \omega\right) \\
& \times \widetilde{f}_{k}\left(\theta_{u} \omega, P_{1}\left(\theta_{u} \omega\right) P_{2}(\omega, u) P_{1}^{-1}(\omega) y\right) d u
\end{aligned}
$$

we obtain that

$$
P\left(\theta_{s} \omega, t\right) \widetilde{g}_{k}\left(s, \omega, t, P^{-1}\left(\theta_{s} \omega, t\right) y\right)=G\left(\theta_{t+s} \omega, y\right) .
$$

The proof finishes by an induction procedure.

Next we detect the tempered variable $R(\omega)$, which is related to the analytic conjugated ball. 
Lemma 21. Let $\rho\left(\theta_{t} \omega\right)$ be a positive tempered function. Assume that the linear cocycle $\Phi(\omega, t)$, continuous in the variable $t$, satisfies the Multiplicative Ergodic Theorem with all zero Lyapunov exponents. Then for any positive $\theta$-invariant function $\tau\left(\theta_{t} \omega\right)=\tau(\omega)$ the following statements hold:

(i) There exists a positive tempered random variable $R(\omega)$ such that $R\left(\theta_{t} \omega\right) \leq \rho\left(\theta_{t} \omega\right)$ and $e^{-\tau(\omega)|s|} R\left(\theta_{t} \omega\right) \leq R\left(\theta_{t+s} \omega\right) \leq e^{\tau(\omega)|s|} R\left(\theta_{t} \omega\right)$.

(ii) There exists a positive tempered random variable $S(\omega)$ such that $e^{-\tau(\omega)|t|} S^{-1}(\omega) \leq\|\Phi(\omega, t)\| \leq e^{\tau(\omega)|t|} S(\omega)$, where $S\left(\theta_{t} \omega\right)$ is tempered and satisfies $e^{-\tau(\omega)|s|} S\left(\theta_{t} \omega\right) \leq S\left(\theta_{t+s} \omega\right) \leq e^{\tau(\omega)|s|} S\left(\theta_{t} \omega\right)$.

Proof: Choosing a monotonically decreasing sequence $\varepsilon_{n} \rightarrow 0$ for each $\tau(\omega) \in\left(\varepsilon_{k+1}, \varepsilon_{k}\right]$, by Proposition 4.3.3 and Theorem 4.3.4(i) in [2], $R_{\varepsilon_{k+1}}(\omega)$ satisfies the conditions of the statement.

In this context we restate Theorem 3 as follows and give the proof out.

Theorem 22. Under hypotheses (i), (ii) and (iii), if the Lyapunov exponents $\gamma(\omega)=\left(\gamma_{1}(\omega), \ldots, \gamma_{d}(\omega)\right)$ have the same signs and are nonresonant, then system (18) can be analytically linearized.

Proof: By the same arguments in the proof of Lemma 20, without loss of generality, we can assume that $\gamma_{i}(\omega) \geq 0$ in system (18) and $q(\omega)=$ $\operatorname{deg}_{x} f\left(\theta_{t} \omega, x\right)>\underline{\gamma}(\omega) / \bar{\gamma}(\omega)$ in system $(22)$. Here $\bar{\gamma}(\omega)=\max _{i}\left\{\gamma_{i}(\omega)\right\}$, $\underline{\gamma}(\omega)=\min _{i}\left\{\gamma_{i}(\bar{\omega})\right\}$ and $q(\omega)=\operatorname{deg}_{x} f(\omega, x)$ means that all the partial derivatives of the function $f$ with respect to the variable $x$ vanish at $x=0$ till the $q(\omega)$-th order.

Next, by setting the variable $\delta(\omega)=(q(\omega) \underline{\gamma}(\omega)-\bar{\gamma}(\omega)) / q(\omega)$, we obtain that $f \in \mathcal{C}_{k \delta}(\Lambda)$ for a fixed $\omega$. Let

$$
\widetilde{f}(s, \omega, t, y)=P^{-1}\left(\theta_{s} \omega, t\right) f\left(\theta_{t+s} \omega, P\left(\theta_{s} \omega, t\right) y\right) .
$$

Thus by hypothesis (ii) we have

$$
\sup _{|y| \leq \rho\left(\theta_{t+s} \omega\right) /\left\|P\left(\theta_{s} \omega, t\right)\right\|}\|\tilde{f}(s, \omega, t, y)\| \leq\left\|P^{-1}\left(\theta_{s} \omega, t\right)\right\| M\left(\theta_{s+t} \omega\right) .
$$

Let $\tau(\omega)=\delta(\omega) / 5$. Note that by Lemma 21 there exist tempered positive functions $R_{1}(\omega), R_{2}(\omega), R_{3}(\omega)$, and $S(\omega)$ such that

$$
\begin{gathered}
\left\|P_{1}\left(\theta_{t+s} \omega\right)\right\| \leq R_{1}\left(\theta_{s+t} \omega\right), \quad\left\|P_{1}\left(\theta_{t+s} \omega\right)^{-1}\right\| \leq R_{2}\left(\theta_{s+t} \omega\right), \\
e^{-\tau(\omega)|t|} S^{-1}\left(\theta_{s} \omega\right) \leq\left\|P_{2}\left(\theta_{s} \omega, t\right)^{ \pm 1}\right\| \leq e^{\tau(\omega)|t|} S\left(\theta_{s} \omega\right)
\end{gathered}
$$

and

$$
\min \left\{\rho\left(\theta_{t} \omega\right), M\left(\theta_{t} \omega\right)^{-1}, 1\right\} \geq R_{3}\left(\theta_{t} \omega\right)
$$


where

$$
\begin{aligned}
& e^{-\tau(\omega)|s|} R_{i}\left(\theta_{t} \omega\right) \leq R_{i}\left(\theta_{t+s} \omega\right) \leq e^{\tau(\omega)|s|} R_{i}\left(\theta_{t} \omega\right), \\
& e^{-\tau(\omega)|s|} S\left(\theta_{t} \omega\right) \leq S\left(\theta_{t+s} \omega\right) \leq e^{\tau(\omega)|s|} S\left(\theta_{t} \omega\right) .
\end{aligned}
$$

Consequently, we can consider

$$
A(s, \omega, t)=\min \left\{\frac{R_{3}\left(\theta_{t+s} \omega\right) S\left(\theta_{s} \omega\right) e^{-\tau(\omega)|t|}}{R_{2}\left(\theta_{s+t} \omega\right)}, \frac{R_{3}\left(\theta_{t+s} \omega\right) S\left(\theta_{s} \omega\right) e^{-\tau(\omega)|t|}}{R_{1}\left(\theta_{s+t} \omega\right)}\right\} .
$$

For fixed $\omega$ and $s$, conditions (11) and (13) are satisfied, thus by Theorem 14 there exist coordinates substitutions $u_{t}=y_{t}+\widetilde{h}(s, \omega, t, y)$ linearizing system (22). Moreover, we have that $\llbracket \widetilde{h} \rrbracket_{r_{0}, A} \leq r_{0}$ for some $r_{0}>0$.

Finally, by Lemma 20 consider $t=0$. Then we obtain $\bar{h}\left(\theta_{s} \omega, y\right)=$ $\widetilde{h}(s, 0, \omega, y)=P_{1}^{-1}\left(\theta_{s} \omega\right) h\left(\theta_{s} \omega, P_{1}\left(\theta_{s} \omega\right) y\right)$, which implies for a fixed $s$ that

$$
\sum_{|k|=1}^{\infty} \sum_{l=1}^{d}\left|\bar{h}_{k}^{l}\left(\theta_{s} \omega\right)\right| A(s, \omega, 0)^{|k|-1} r_{0}^{|k|} \leq \llbracket \widetilde{h} \rrbracket_{r_{0}, A} \leq r_{0} .
$$

That is, $\bar{h}(\omega, y)$ is analytic in the tempered ball $|y|<A(s, \omega, 0)$. Hence, it is enough to show the analyticity of $\widetilde{h}$ in $|y| \leq A(0, \omega, 0) /\left\|P_{1}(\omega)\right\|$. Obviously $x \mapsto x+h\left(\theta_{t} \omega, x\right)$ changes system (18) into its linear part and the proof is complete.

\section{Almost periodic systems}

In this part, we deal with the analytic linearization for almost periodic systems, which obey averaging laws.

First of all, we do simplifications to the linear part. Consider the following linear differential system

$$
\dot{x}=\varepsilon(A+\widetilde{A}(t, \varepsilon)) x,
$$

where $\widetilde{A}(t, \varepsilon) \rightarrow 0$ as $\varepsilon \rightarrow 0$ uniformly for $t \in \mathbb{R}$ and it is almost periodic in the variable $t$. Assume that $A$ is in the Jordan normal form. Then by Lemma 7 building a change of variables $x=(E+H(t, \varepsilon)) y$ the original system can be changed into

$$
\dot{y}=\varepsilon(A+\widehat{A}(t, \varepsilon)) y .
$$

Here $\widehat{A}$ is in the block diagonal form with respect to the different real parts of eigenvalues, $\widehat{A}(t, \varepsilon) \rightarrow 0$ as $\varepsilon \rightarrow 0$ uniformly for $t \in \mathbb{R}$ and 
$m(\widehat{A}) \subseteq m(\widetilde{A})$. Now we force the linear system into a constant and diagonal one. Of course, such transformations generally cannot be almost periodic in $t$.

Lemma 23. Doing the change of variables $y=\widehat{H}(t, \varepsilon) z$, system $(23)$ can be change into

$$
\dot{z}=\varepsilon \Lambda z,
$$

where the matrix $\Lambda=\operatorname{diag}\left(\operatorname{Re} \lambda_{1}, \ldots, \operatorname{Re} \lambda_{d}\right)$ with $\lambda(A)=\left(\lambda_{1}, \ldots, \lambda_{d}\right)$. For any $\tau>0$, there exist a positive constant $\mu$ and a function $\rho(t)$ such that $\max \left\{|\widehat{H}(t, \varepsilon)|,\left|\widehat{H}^{-1}(t, \varepsilon)\right|\right\} \leq \rho(t)$ for fixed $t$ and $|\varepsilon| \leq \mu$ and

$$
\frac{\rho(t+h)}{\rho(t)} \leq e^{\varepsilon \tau|h|}, \quad t, h \in \mathbb{R} .
$$

Proof: Without loss of generality, we take system (23) into account. Let $\Upsilon=\operatorname{diag}\left(\operatorname{Im} \lambda_{1}, \ldots, \operatorname{Im} \lambda_{d}\right)$ and $N$ be the nilpotent part. Then $A=\Lambda+\sqrt{-1} \Upsilon+N$. Since $\widehat{A}$ is in the block diagonal form with respect to $\Lambda$, it implies $\widehat{A} \Lambda=\Lambda \widehat{A}$. Let $\widehat{H}(t, \varepsilon)$ be the fundamental matrix solution of the following system

$$
\dot{y}=\varepsilon(\sqrt{-1} \Upsilon+N+\widehat{A}(t, \varepsilon)) y .
$$

This is the desired transformation. At last, for that $\tau$ we can find $\mu>0$ such that $\|\widehat{A}\| \leq \tau / 4$ for $|\varepsilon| \leq \mu$. Thus the equation

$$
\dot{y}=\varepsilon(\sqrt{-1} \Upsilon+N+\widehat{A}(t, \varepsilon)-\tau / 2) y,
$$

whose fundamental matrix solution is remarked as $\Psi(t, \varepsilon, \tau)$, is positive asymptotically stable uniformly for any initial time $t_{0} \in \mathbb{R}$. Now consider

$$
K_{1}=\sup _{t \geq 0,|\varepsilon| \leq \mu}\left\{|\Psi(t, \varepsilon, \tau)|,\left|\Psi^{-1}(t, \varepsilon, \tau)\right|\right\} .
$$

It admits $|\widehat{H}(t, \varepsilon)| \leq K_{1} e^{\varepsilon \tau t / 2}$ and $\left|\widehat{H}^{-1}(t, \varepsilon)\right| \leq K_{1} e^{\varepsilon \tau t / 2}$ for $t \geq 0$ and $|\varepsilon|<\mu$. Similarly, for

$$
\dot{y}=\varepsilon(\sqrt{-1} \Upsilon+N-\widehat{A}(t, \varepsilon)) y,
$$

we can get

$$
K_{2}=\sup _{t \leq 0,|\varepsilon| \leq \mu}\left\{|\widehat{\Psi}(t, \varepsilon, \tau)|,\left|\widehat{\Psi}^{-1}(t, \varepsilon, \tau)\right|\right\},
$$

where $\widehat{\Psi}^{-1}(t, \varepsilon, \tau)$ is the corresponding fundamental matrix solution. Taking $\rho(t)=\left(K_{1}+K_{2}\right) e^{\varepsilon \tau|t| / 2}$ the proof is finished.

Next we show that any formal transformation fulfilling Theorem 4 with uniformly bounded Taylor coefficient functions is almost periodic. 
Lemma 24. Under the conditions of Theorem 4, if the formal transformation $w=\sum_{k, j} w_{k, j}(t, \varepsilon) z^{k} e_{j}$ exists, satisfying $\sup _{t \in \mathbb{R}}\left|w_{k, j}\right|<\infty$ and turning system (6) into its linear part, then $w_{k, j}$ is almost periodic in $t$ and $m\left(w_{k, j}\right) \subseteq m(F)$ for a sufficiently small $\varepsilon$.

Proof: Without loss of generality, we can assume that $\operatorname{Re} \lambda_{1} \geq \operatorname{Re} \lambda_{2} \geq$ $\cdots \geq \operatorname{Re} \lambda_{d}>0$ for $\lambda(A)=\left(\lambda_{1}, \ldots, \lambda_{d}\right)$. Let

$$
F(t, \varepsilon, y)=(A+\widehat{A}(t, \varepsilon)) y+\sum_{k, j} F_{k, j}(t, \varepsilon) y^{k} e_{j}
$$

and $\widehat{F}=F-(A+\widehat{A}(t, \varepsilon)) y$. Of course, as it is shown in formula (10) $w$ satisfies

$$
\operatorname{ad}_{\varepsilon(A+\widehat{A})} w(t, \varepsilon, y)=\mathrm{S}_{\varepsilon \widehat{F}} w(t, \varepsilon, y) .
$$

Since $\widehat{A} \rightarrow 0$ as $\varepsilon \rightarrow 0$ uniformly for $t \in \mathbb{R}$, so does the operator ad, i.e. $\operatorname{ad}_{\varepsilon(A+\widehat{A})} \rightarrow \operatorname{ad}_{\varepsilon A}$ as $\varepsilon \rightarrow 0$ uniformly for $t \in \mathbb{R}$. Let $\operatorname{ad}_{A}=\partial_{t}+L_{A}$, where $L_{A} h:=D h A y-A h$ is the same as the one used for autonomous systems. Then by the classical normal form theory, the eigenvalues set of $L_{\varepsilon A}$ is

$$
\left\{\varepsilon\left(\langle k, \lambda\rangle-\lambda_{j}\right)|| k \mid \geq 2, j=1, \ldots, d\right\} .
$$

So for $|k|=2$, the corresponding equations admit exponential dichotomy for a sufficiently small $\varepsilon$ under the non-resonant condition by Lemma 7 . Using Lemma 8, we get the desired almost periodic solution. At last, by an induction procedure, the proof is completed.

Finally we prove Theorem 4 .

Proof of Theorem 4: Let's begin with system (6). Without loss of generality, by Lemma 7 we can consider

$$
F(t, \varepsilon, y)=(A+\widehat{A}(t, \varepsilon)) y+\sum_{k, j} F_{k, j}(t, \varepsilon) y^{k} e_{j} .
$$

Here $A$ is in the Jordan normal form and $\widehat{A}$ is block diagonal with respect to the different real parts of the eigenvalues of $A$. Then $\widehat{F}=F-(A+$ $\widehat{A}(t, \varepsilon)) y$ contains all higher order terms.

Here we consider the following system

$$
\dot{z}=\varepsilon \Lambda z+\varepsilon \widehat{H}^{-1}(t, \varepsilon) \widehat{F}(t+s, \varepsilon, \widehat{H}(t, \varepsilon) z),
$$

where $\widehat{H}$ is the same given in Lemma 23. So the function $\breve{F}=\widehat{H}^{-1}(t, \varepsilon) \widehat{F}(t+s, \varepsilon, \widehat{H}(t, \varepsilon) z)$ satisfies

$$
\sup _{s \in \mathbb{R},|z| \leq r_{1} \rho^{-1}(t)}|\breve{F}| \leq \rho(t)|| \widehat{F}|| \leq K \rho(t), \quad|\varepsilon|<\mu_{1},
$$


where $\rho(t)$ is also given in Lemma 23 and $K=2 \sup _{t \in \mathbb{R}, z \in U_{r_{1}}}|\widehat{F}(t, 0, z)|$. Let $\widehat{\lambda}=\max \left\{\operatorname{Re} \lambda_{i}\right\}$ and $\breve{\lambda}=\min \left\{\operatorname{Re} \lambda_{i}\right\}$. Thus, when all $\operatorname{Re} \lambda_{i}$ are positive, we have that $\langle k, \operatorname{Re} \lambda\rangle-\operatorname{Re} \lambda_{j} \geq \delta^{\prime}|k|$, where $\delta^{\prime}=\breve{\lambda}-\widehat{\lambda} / \widehat{d}$ and $\widehat{d}$ is a positive integer satisfying $\widehat{d}>\widehat{\lambda} / \breve{\lambda}$. Similarly when all $\operatorname{Re} \lambda_{i}$ are negative, we have $\langle k, \operatorname{Re} \lambda\rangle-\operatorname{Re} \lambda_{j} \leq-\delta^{\prime}|k|$ instead, where $\delta^{\prime}=-\widehat{\lambda}+\breve{\lambda} / \widehat{d}$ and $\widehat{d}>\breve{\lambda} / \widehat{\lambda}$. Notice that by the proof of Lemma 24 all higher order terms, whose degree is less than $\widehat{d}$, can be removed. So all the conditions of Theorem 1 are well prepared for $\breve{F} \in \mathcal{C}_{\varepsilon k \delta^{\prime}}(\varepsilon \Lambda)$.

Since here a clear estimation of the changes is necessary, we apply Lemma 13 instead. So the linearized transformation $\widetilde{H}$ is a fixed point in $\left(B_{r}, \square \cdot \square_{r, \kappa}\right)$ with $\kappa(t)=\min \left\{r_{1} \rho^{-1}(t), K^{-1} \rho^{-1}(t), 1\right\}$. Moreover, note that in Lemma 10 the bounded operator norm is given by

$$
\left\|\operatorname{ad}_{\varepsilon \Lambda}^{-1}\right\| \leq \frac{2}{\varepsilon \delta^{\prime}}
$$

after using $\tau=\delta^{\prime} / 2$ in Lemma 23, where $\|\cdot\|$ is the operator norm in $\left(B_{r}, \square \cdot \square_{r, \kappa}\right)$. Meanwhile, the strong contraction of the operator $\mathrm{S}_{\varepsilon \breve{F}}$ in Lemma 13 is measured by

$$
\left\|D_{z}(\varepsilon \breve{F})\right\|_{3 r, \kappa} \leq C \varepsilon r .
$$

Therefore, the contraction property of the composition operator $\operatorname{ad}_{\varepsilon \Lambda}^{-1} \circ \mathrm{S}_{\varepsilon \breve{F}}$ remains.

Finally, in Lemma 20 setting the group action $\theta_{t} \cdot=t+\cdot$, we get that the solution formally can be written as

$$
\widetilde{H}(t, s, \varepsilon, y)=\widehat{H}^{-1}(t, \varepsilon) \breve{H}(\widehat{H}(t, \varepsilon) z, t+s, \varepsilon) .
$$

Hence, $\breve{H}(s, \varepsilon, y)=\widetilde{H}(0, s, \varepsilon, y)$ is what we seek, which satisfies the condition $\llbracket \breve{H} \rrbracket_{r_{0} \kappa(0)} \leq r_{0} \kappa(0)$ and it is almost periodic in $s$ by Lemma 24 . This completes the proof.

\section{Other applications}

In this last part we first apply the majorant norm method to prove a kernel result in $[\mathbf{3}]$, which has originally been solved by computing the holomorphy. Then, we show a result of [18] in the study of the integrability of the Lotka-Volterra systems.

\section{Analytic conjugacy with the parameter.}

Consider the parametric analytic system

$$
\dot{x}=D(s) x+f(s, x)=F(s, x),
$$


where $s \in \mathbb{C}^{m}$ is a complex parameter, $x \in \mathbb{C}^{d}, F$ is holomorphic in $(0,0) \in \mathbb{C}^{m+d}$ and $D(s)=\operatorname{diag}\left(\lambda_{1}(s), \ldots, \lambda_{d}(s)\right)$. When $D(s)=$ $\operatorname{diag}\left(\lambda_{1}(s), \ldots, \lambda_{d}(s)\right)$ is diagonal and $s$ is a parameter, then we say $f \in$ $\mathcal{C}_{\delta}(D(s))$ associated with the nonlinearity $f(s, x)=\sum_{|k| \geq 2} \sum_{l=1}^{d} f_{k}^{l}(s) x^{k} e_{l}$, provided that there exists $\delta>0$ such that $\sum_{i=1}^{d} \lambda_{i}(s) k_{i}-\lambda_{l} \geq \delta$ or $\sum_{i=1}^{d} \lambda_{i}(s) k_{i}-\lambda_{l} \leq-\delta$ uniformly for $|s| \leq r$ and all the monomial $\sim x^{k} e_{l}$ in the expansion series of $f$. Similarly, the operators $\operatorname{ad}_{D}$ and $\mathrm{S}_{f}$ can be defined by $\operatorname{ad}_{D}: h(s, x) \mapsto D_{x} h(s, x) D(s) x-D h(s, x)$ and $\mathrm{S}_{f}: h(s, x) \mapsto f(s, x+h(s, x))$. The arguments are similar except the following.

Lemma 25. If $f$ and $h \in \mathcal{C}_{\delta}(D(s))$, then we have that $\mathrm{S}_{f}(h) \in \mathcal{C}_{\delta}(D(s))$.

Proof: Without loss of generality, we denote the coefficients, whatever depending on $s$ or not, simply as $\sim$. So $f=\sum \sim x^{k} e_{l}$ for $\langle k, \lambda\rangle-\lambda_{l} \leq-\delta$.

We have that

$$
\begin{aligned}
f(s, x+h(s, x)) & =\sum f_{k}^{l}(s)(x+h(s, x))^{k} e_{l} \\
& =\sum \sim \prod_{\alpha=1}^{d}\left(\sim x_{\alpha}+\sum_{\mu} \sim x^{m_{\alpha \mu}}\right)^{k_{\alpha}} e_{l} \\
& =\sum \sim\left(\prod_{\alpha=1}^{d} x_{\alpha}^{i_{\alpha 0}}\right) x^{\sum_{\alpha=1}^{d} \sum_{\mu \geq 1} i_{\alpha \mu} m_{\alpha \mu}} e_{l},
\end{aligned}
$$

where $k=\left(k_{1}, \ldots, k_{d}\right), \sim x^{m_{\alpha \mu}} e_{\alpha}$ is a monomial of $h$ with the non-zero coefficient and $\sum_{\mu=0}^{\infty} i_{\alpha \mu}=k_{\alpha}$ for $\alpha=1, \ldots, d$. Thus we have that

$$
\begin{aligned}
& -\lambda_{l}+\sum_{\alpha=1}^{d} i_{\alpha 0} \lambda_{\alpha}+\sum_{\alpha=1}^{d} \sum_{\mu \geq 1}\left\langle i_{\alpha \mu} m_{\alpha \mu}, \lambda\right\rangle \\
& \leq-\lambda_{l}+\sum_{\alpha=1}^{d} i_{\alpha 0} \lambda_{\alpha}+\sum_{\alpha=1}^{d} \sum_{\mu \geq 1} i_{\alpha \mu}\left(-\delta+\lambda_{\alpha}\right) \\
& =-\lambda_{l}+\langle k, \lambda\rangle-\delta \sum_{\alpha=1}^{d} \sum_{\mu \geq 1} i_{\alpha \mu} \leq-\delta .
\end{aligned}
$$

This completes the proof.

Set $Q_{r}=\left\{h \mid h(s, 0)=0, \square h \rrbracket_{r} \leq r, h \in \mathcal{C}_{\delta}(D(s))\right\}$. Now we reprove Theorem 3 in $[\mathbf{3}]$ by majorant norm methods. 
Proposition 26. If $f \in \mathcal{C}_{\delta}(D(s))$, then system (24) can be linearized by the coordinates substitutions $y=x+h(s, x)$, which is holomorphic at the origin in $\mathbb{C}^{m+d}$.

Proof: First, it is easy to verify that

$$
\left(\operatorname{ad}_{D}\right)^{-1}: f(s, x)=\sum f_{k}^{l}(s) x^{k} e_{l} \mapsto \sum \frac{f_{k}^{l}(s) x^{k} e_{l}}{\langle\lambda(s), k\rangle-\lambda_{l}(s)}
$$

So $\square\left(\operatorname{ad}_{D}\right)^{-1} f \rrbracket_{r} \leq \llbracket f \square / \delta$ is uniformly bounded in $Q_{r}$.

Next, Lemma 13 is still valid if we take $\kappa(t)=r$, which means that $\mathrm{S}_{f}$ is strongly contractive when $r$ is small enough. Moreover, taking $\sup _{|s| \leq w,|x| \leq w}|f(s, x)| \leq M$, by Cauchy estimation, we obtain that $\sup _{|s| \leq w}\left|f_{k}^{l}(s)\right| \leq M / w^{|k|}$, which leads to

$$
\square D_{x} f \rrbracket_{r} \leq d \sum_{k, l}\left|f_{k}^{l}(s)\right||k| r^{|k|} \leq r M d \sum_{|k| \geq 0}|k|(1 / 2)^{|k|}
$$

for $r<w / 2$. Consequently, the Lipschitz constant is independent of $s$.

The proof finishes using similar arguments like in Theorem 14. The composed operator $\operatorname{ad}_{D}^{-1} \circ \mathrm{S}_{f}(h)=h$ has a unique fixed point with $\llbracket h \rrbracket_{r_{0}} \leq r_{0}$ via the Contracting Mapping Principle, which also implies the analyticity with respect to $s$.

\section{Integrability of the Lotka-Volterra systems.}

The integrability problem for the $1:-q$ resonant singular point is attractive. See [18] for more introductions. Here we just provide uniform proofs for Proposition 3.1, Theorem 3.1 and 3.2 in [18] together.

Consider the quadratic differential system

$$
\dot{x}=x+a x^{2}+b x y+f y^{2}, \quad \dot{y}=-\lambda y+c x y+d y^{2} .
$$

Proposition 27. Let $\lambda>0$. If $c / a+\lambda=k \in \mathbb{N}^{+}$with $2 \leq k<\lambda+1$, then system (25) is analytically integrable.

Proof: The transformation $(u, v)=\left(a x, y(1+a x)^{-k}\right)$ changes system $(25)$ to

$$
\begin{aligned}
& \dot{u}=\left(u+b u(1+u)^{k-1} v+a f(1+u)^{2 k-1} v^{2}\right)(1+u), \\
& \dot{v}=\left(-\lambda v+d(1+u)^{k-1} v^{2}-k b u(1+u)^{k-1} v^{2}-k a f(1+u)^{2 k-2} v^{3}\right)(1+u),
\end{aligned}
$$


which is orbitally equivalent to

$$
\begin{aligned}
& \dot{u}=u+b u(1+u)^{k-1} v+a f(1+u)^{2 k-1} v^{2}, \\
& \dot{v}=-\lambda v+d(1+u)^{k-1} v^{2}-k b u(1+u)^{k-1} v^{2}-k a f(1+u)^{2 k-2} v^{3} .
\end{aligned}
$$

Let $\delta=k-1-\lambda<0$. Then Proposition 26 can be applied and the proof is complete.

Acknowledgements. The author is supported by NSFC for Young Scientists of China (no. 11001047) and partially supported by NSF of Jiangsu, China (no. BK2010420).

\section{References}

[1] L. YA. AdRIANova, "Introduction to linear systems of differential equations", Translated from the Russian by Peter Zhevandrov, Translations of Mathematical Monographs 146, American Mathematical Society, Providence, RI, 1995.

[2] L. Arnold, "Random dynamical systems", Springer Monographs in Mathematics, Springer-Verlag, Berlin, 1998.

[3] J. Basto-Gonçalves and I. Cruz, Analytic linearizability of some resonant vector fields, Proc. Amer. Math. Soc. 129(8) (2001), 2473-2481 (electronic). DOI: 10.1090/S0002-9939-00-05796-8.

[4] Y. N. BiBikov, "Local theory of nonlinear analytic ordinary differential equations", Lecture Notes in Mathematics 702, SpringerVerlag, Berlin-New York, 1979.

[5] K.-T. Chen, Equivalence and decomposition of vector fields about an elementary critical point, Amer. J. Math. 85 (1963), 693-722.

[6] W. A. Coppel, "Dichotomies in stability theory", Lecture Notes in Mathematics 629, Springer-Verlag, Berlin-New York, 1978.

[7] R. D. Costin, Analytic linearization of nonlinear perturbations of Fuchsian systems, Nonlinearity 21(9) (2008), 2083-2097. DOI: 10.1088/0951-7715/21/9/010.

[8] R. D. Costin, Nonlinear perturbations of Fuchsian systems: corrections and linearization, normal forms, Nonlinearity 21(9) (2008), 2073-2082. DOI: 10.1088/0951-7715/21/9/009.

[9] A. M. Fink, "Almost periodic differential equations", Lecture Notes in Mathematics 377, Springer-Verlag, Berlin-New York, 1974. 
[10] J. K. Hale, "Ordinary differential equations", Second edition, Robert E. Krieger Publishing Co., Inc., Huntington, N.Y., 1980.

[11] Ju. S. IL'JAŠENKO, Divergence of series that reduce an analytic differential equation to linear normal form at a singular point, (Russian), Funktsional. Anal. i Prilozhen. 13(3) (1979), 87-88; translation in: Functional Anal. Appl. 13(3) (1979), 227-228 (1980).

[12] Yu. S. Il'yashenko and S. Yu. Yakovenko, Finitely smooth normal forms of local families of diffeomorphisms and vector fields, (Russian), Uspekhi Mat. Nauk 46(1) (1991), 3-39, 240; translation in: Russian Math. Surveys 46(1) (1991), 1-43. DOI: 10.1070/RM1991v046n01ABEH002733.

[13] Yu. Ilyashenko And S. Yu. YAKOVEnKo, "Lectures on analytic differential equations", Graduate Studies in Mathematics 86, American Mathematical Society, Providence, RI, 2008.

[14] L. Jiao, M. Li, AND H. Wu, General laws of the analytic linearization for random diffeomorphisms, Math. Z. 270(3-4) (2012), 739-757. DOI : 10.1007/s00209-010-0824-0.

[15] W. Li, J. Llibre, AND H. Wu, Polynomial and linearized normal forms for almost periodic difference systems, J. Difference Equ. Appl. 15(10) (2009), 927-948. DOI: 10.1080/10236190802284848.

[16] W. Li AND K. LU, Poincaré theorems for random dynamical systems, Ergodic Theory Dynam. Systems 25(4) (2005), 1221-1236. DOI: $10.1017 /$ S014338570400094X.

[17] W. LI AND K. LU, Sternberg theorems for random dynamical systems, Comm. Pure Appl. Math. 58(7) (2005), 941-988. DOI: 10.1002/сра. 20083.

[18] C. LiU, G. Chen, And C. Li, Integrability and linearizability of the Lotka-Volterra systems, J. Differential Equations 198(2) (2004), 301-320. DOI : 10.1016/S0022-0396(03)00196-7.

[19] H. Poincaré, Thesis (1879); also: "Evres de Henri Poincaré", Publiées sous les auspices de l'Académie des Sciences par P. Appell. T. I. Publié avec la collaboration de J. Drach, Gauthier-Villars, Paris, 1928.

[20] S. SternberG, Infinite Lie groups and the formal aspects of dynamical systems, J. Math. Mech. 10 (1961), 451-474.

[21] F. TAKEns, Normal forms for certain singularities of vector fields, Colloque International sur l'Analyse et la Topologie Différentielle (Colloques Internationaux du Centre National de la Recherche Scientifique, Strasbourg, 1972), Ann. Inst. Fourier (Grenoble) 23(2) (1973), 163-195. 
[22] H. Wu And W. Li, Poincaré type theorems for non-autonomous systems, J. Differential Equations 245(10) (2008), 2958-2978. DOI: 10.1016/j.jde.2008.05.005.

Department of Mathematics

Southeast University

210096 Nanjing, Jiangsu

China

E-mail address: haow@seu.edu.cn 